\section{P4.35 REDUCING HIV RISK BEHAVIOUR AFTER INTERVENTION WITH YOUNG LEADERS OF KEY POPULATIONS IN BRAZIL}

Diego Agostinho Calixto, Alicia Kruger, Carina Bernardes, Gilvane Casimiro da Silva, Paula Emilia Adamy, Adele Schwartz Benzaken. Ministry of Health of Brazil - Department of STI, HIVIAIDS and Viral Hepatitis, Brasilia, Brazil

\subsection{6/sextrans-2017-053264.532}

Introduction Peer dialogues influence the adoption of behavioural changes to reduce the risk of HIV infection (human immunodeficiency virus). By intervening experimentally in the community to change risk behaviour patterns, it may be possible to promote widespread reductions in HIV risk practices within a population.

Methods The intervention identified and trained young age range 18-26 people who are reliably identified as leaders among one of these key populations - gay men and MSM, transgender people (transvestites and transsexual women), drug users and harm reducers and sex workers - in all five regions of Brazil to act as multipliers of behavioural changes for their peers, in relation to HIV. We also include young people living with HIV, considering that it is important that these young people share the experience of living with HIV with other young people in greater vulnerability and risk.

Results 140 young people from the key populations were trained in the 5 Brazilian regions. The proportions of the key populations trained in this intervention were 41.9 per cent homosexuals and MSM, 14.5 per cent harm reduction or drug users, 8 per cent transgender people, 6 per cent sex workers and 15 per cent young people living With HIV. Approximately $70 \%$ of young trained in this intervention have already developed some activity to multiply the information about prevention and behavioural practices to reduce HIV infection in their respective territories and communities, promoting knowledge about combination prevention and changes related to sexual practices and behaviours.

Conclusion Interventions that empower young to endorse change can produce or accelerate changes in the behaviour and sexual practices of the young population to reduce the risk of HIV infection. These interventions have developed a network of multipliers in a successive chain of HIV information and combination prevention to reduce HIV infection and risk behaviours through peer-to-peer communication at the community level among young key populations in Brazil.

\section{P4.36 EVALUATING THE COSTS, BENEFITS AND COST- EFFECTIVENESS OF MULTI-PATHOGEN POINT-OF-CARE TESTS FOR SEXUALLY TRANSMITTED INFECTIONS IN SYMPTOMATIC GENITOURINARY MEDICINE CLINIC ATTENDEES}

${ }^{1}$ Emma Harding-Esch, ${ }^{2}$ Susie E Huntington, ${ }^{2}$ Richeal M Burns, ${ }^{2}$ Mike Harvey, ${ }^{3}$ Rachel HillTout, 'Sebastian S Fuller, ${ }^{2}$ Elisabeth J Adams, 'S Tariq Sadiq. 'Applied Diagnostic Research and Evaluation Unit, St George's University of London, London, UK; ${ }^{2}$ Aquarius Population Health, London, UK; ${ }^{3}$ St George's University Hospitals NHS Foundation Trust, London, UK

\subsection{6/sextrans-2017-053264.533}

Introduction In sexual health services, availability of rapid and accurate point-of-care tests (POCTs) may enable major improvements in care pathway efficiencies and outcomes. Previous economic evaluations of nucleic acid amplification test (NAAT) POCTs for Chlamydia trachomatis (CT) and Neisseria gonorrhoeae (NG) indicate they may provide a cost-effective strategy for screening genitourinary medicine (GUM) attendees. We estimated costs, benefits and cost-effectiveness of three strategies using accurate, rapid NAAT POCTs that could detect different combinations of common multiple sexually transmitted infections (mSTIs) compared with standard care (SC; laboratory-based CT/NG NAAT).

Methods A decision tree was constructed to simulate a hypothetical cohort of 965988 patients, representing annual numbers of GUM attendees in England, symptomatic for lower genitourinary tract infection. The model considered delivery costs (micro-costing) and reimbursement (tariff) to GUM services associated with diagnosing and managing STIs. POCT strategies compared to SC were: A) POCT for CT and NG; B) POCT for CT-NG and Mycoplasma genitalium (MG); C) POCT for CT-NG-MG and Trichomonas vaginalis. Data came from published literature and unpublished estimates.

Results SC was cheaper than all POCT strategies when microcosting, but POCT $\mathrm{C}$ was the cheapest strategy for tariff costings. POCT C's incremental cost-effectiveness ratio (ICER) was $£ 36585$ per quality-adjusted life year (QALY) gained compared to SC when micro-costing; it was cost-saving (by $£ 26,451,382$ ) when tariff costing was applied. POCT C also generated most benefits, with 240467 fewer clinic attendances, 808 fewer onward STI transmissions, and 235135 averted inappropriate treatments compared to SC.

Conclusion POCTs that detect STI diagnoses may be costeffective, cost-saving and improve patient management. However, there is variation by costing strategy, patient population, clinical setting and patient pathways. Further evidence is needed to populate model parameters to reduce uncertainty in economic analyses.

\section{P4.37 THE PERCEPTION AND DETERMINANTS OF SEXUAL BEHAVIOUR OF UNIVERSITY UNDERGRADUATES AT A TERTIARY INSTITUTION IN NIGERIA - WHAT ARE THE UNDERLYING FACTORS}

${ }^{1}$ Emmanuel Shobowale, ${ }^{1} \mathrm{O}$ Abiodun, ${ }^{1} \mathrm{CJ}$ Elikwu, ${ }^{2} \mathrm{KI}$ Onyedibe, ${ }^{1} \mathrm{~J}$ Sotunsa, ${ }^{3} \mathrm{O}$ lyoha. ${ }^{1}$ Babcock University, Ogun, Nigeria; ${ }^{2}$ University of Jos, Plateau, Nigeria; ${ }^{3}$ University of Benin, Benin, Nigeria

\subsection{6/sextrans-2017-053264.534}

Introduction Sexually Transmitted Infections are widespread, and have effects on the reproductive and sexual health of the general population particularly youths and adolescents. The study was done to assess the level of knowledge, perception and attitudes towards STI's by undergraduate students. The objective was to understand and describe the drivers of sexual behaviour amongst university undergraduates and gain an insight into their perception of sexually transmitted infections. Methods This was a cross-sectional study. A structured selfadministered questionnaire on risk factors for STI's was given to respondents to answer. The study was conducted in October 2016.

Results The mean age of the students $(n=310)$ was 17.8 years +/-1.77 SD.: males were 0.39 times more likely to be sexually active than females $\left[\mathrm{p}=<0.001, \mathrm{X}^{2}=15.0, \mathrm{CI}=0.23-0.69\right]$, females were 2.18 times more likely to join an abstinence club $\left[\mathrm{p}=0.005, \mathrm{X}^{2}=7.6, \mathrm{CI}=1.24-3.81\right]$. Males were 0.3 times more likely to believe condoms protect against all STI's [p $\left.=<0.001, \mathrm{X}^{2}=10.93, \mathrm{CI}=0.14-0.63\right]$ and were 2.61 times 
more likely to use condoms regularly $\left[\mathrm{p}=0.002, \mathrm{X}^{2}=8.94\right.$, $\mathrm{CI}=1.38-4.94]$.

Conclusion The rates of sexual exposure and unprotected sex are still high among our youths. We need to invest in treatment and prevention programs regarding STI's in the young and identify the barriers that prevent access to care such as a lack of appropriate and effective STI control programs. STIs are preventable and significant reductions in new infections are possible and needed. Prevention can minimise their negative impact and reduce healthcare costs.

\section{P4.38 "I MET HIM ON JACK'D” - EXPLORING HIV RISK PERCEPTIONS AND RISK MITIGATION STRATEGIES OF YOUNG BLACK MSM MEETING SEX PARTNERS ON GEOSOCIAL NETWORKING APPS"}

Errol L Fields, Anthony Morgan, Renata Arrington-Sanders, Jacky M Jennings. Johns Hopkins University School of Medicine, Baltimore, USA

\subsection{6/sextrans-2017-053264.535}

Introduction Young Black MSM (YBMSM) continue to experience HIV disparities in the US. Increasingly evidence suggests these disparities are perpetuated by exposure to sexual networks with higher HIV incidence and untreated prevalence. In Baltimore, there is high use of geosocial networking (GSN) apps to meet sex partners among newly diagnosed HIVinfected YBMSM, yet little is known about how they navigate these potentially risky environments. We interviewed app users to explore perceptions of and strategies to mitigate HIV risk in these spaces.

Methods We actively recruited YBMSM $(n=17)$ age 18-24 $($ mean $=21.5 / \mathrm{SD}=1.8$ ) from the most frequently reported GSN-app among new MSM HIV cases in Baltimore. Participants were recruited by direct messaging while logged-on in high HIV transmission venues or census tracts identified using surveillance and community viral load data. Participants completed 60-90 min in-depth interviews which were audiorecorded and transcribed. Transcripts were analysed using categorical analysis including a 3 -stage analytic coding strategy and were double-coded until consistency was achieved.

Results Participants perceived a high risk of acquiring HIV from partners met on the app but also described strategies they felt mitigated those risk. Some participants described direct sexual health communication with potential partners often involving a discussion of HIV status. A second more commonly described strategy was to presume a potential partner was safe or not safe based on social cues or profile characteristics. The third strategy involved limiting numbers of partners or limiting sex to activities believed to involve less HIV risk.

Conclusion Sexual networks are an important driver of HIV risk among YBMSM and GSN apps are increasingly becoming a central node in these networks. We found that young men are aware of their risk while navigating these social spaces, but may be using ineffective strategies to mitigate those risk. Future research examining strategies to reach youth in these spaces to help them make healthier sexual connexions is needed.

\section{P4.39 EFFECTIVENESS OF PEER EDUCATION INTERVENTIONS FOR STIS PREVENTION AMONG FEMALE PRISONERS IN NORTHEAST OF IRAN}

Farzad Jalali, Alireza Hasani, Niloofar Fakoor Sharghi, Seyedeh Fatemeh Hashemi. Negahe Mosbat Social Health Institute, Mashhad, Iran

\subsection{6/sextrans-2017-053264.536}

Introduction Sexually Transmitted Infections (STDs) have become an important medical problem in prisons all around the world. Peer education interventions are a frequently utilised strategy for preventing Sexually Transmitted Infections (STIs) worldwide. Our study was aimed to examine the effectiveness of the peer education method in knowledge, attitudes, and practices, regarding to STIs.

Methods A peer education intervention was conducted in a female prison in the northeast of Iran. A cohort of 1098 students was surveyed ( $n=534$ intervention group, $n=567$ control group) through anonymous questionnaires, both pre- and postintervention.

Results There were significant differences over time and between intervention and control groups associated with increased STIs knowledge (OR:2.16; 95\% CI:1.76, 2.23), reduced equipment sharing among injection drug users (OR:0.43; 95\% CI:0.20, 0.52), and increased condom use (OR:2.23; 95\% CI:1.69, 2.43). Peer education programs had a significant effect on STI infection (OR: 2.26; 95\% CI:1.99, 2.16).

Conclusion Peer education programs in a female prison are effective at improving behavioural, practical and infection outcomes regarding STIs. According to high prevalence of STIs among female inmates, prevention activities targeting this population should be considered.

\section{P4.40 THE EFFECTIVENESS OF COGNITIVE GROUP THERAPY BASED ON SCHEMA-FOCUSED APPROACH FOR DECREASING DEPRESSION IN PRISONERS LIVING WITH HIV}

Farzad Jalali, Alireza Hasani, Niloofar Fakoor Sharghi, Seyedeh Fatemeh Hashemi. Negahe Mosbat Social Health Institute, Mashhad, Iran

\subsection{6/sextrans-2017-053264.537}

Introduction Studies have shown that about half of HIV-positive people are involved in a variety of psychiatric disorders which depression is the most common. The chance of developing a depressive disorder is two times higher in HIVinfected patients than in HIV negative comparison subjects. Mental disorders are over-represented in prisoners. The aim of this study was to determine the effectiveness of cognitive group therapy based on schema-focused approach in decreasing depression in prisoners living with HIV.

Methods The design of this study was semi-experimental with pre-test, post-test and control group. The research population consisted of all prisoners living with HIV in Mashhad Central Prison. 20 prisoners living with HIV were selected by convenience sampling, and randomly assigned to an experimental group (10 prisoners) and a control group (10 prisoners). The experimental group received schema-focused cognitive group therapy while the control group received no treatment. The research measurement instruments consisted of SQ-SF and BDI-II. ANCOVA models were used to test the study hypothesis. 
Results Cognitive group therapy based on schema focused approach decreased maladaptive schemas in the experimental group compare to the control group. Subsequently depression was decreased in the experimental group compare to the control group.

Conclusion This study showed that Schema Therapy is an effective treatment for depression in special populations. Life of people living with HIV, particularly in prisons, is full of emotional deprivation, defects and damage; these reasons can cause conditions which is lead to depression. ST through modulation maladaptive schemes could reduce depression in prisoners living with HIV.

\section{P4.41 EFFECT OF HIV RELATED STIGMA ON UTILISATION OF SKILLED BIRTH ATTENDANTS BY HIV POSITIVE WOMEN IN NIGERIA; A SYSTEMATIC REVIEW}

Foluso Ishola. Japan International Cooperation Agency, Lagos, Nigeria

\subsection{6/sextrans-2017-053264.538}

Introduction HIV/AIDS-related stigma is recognised as a major barrier to utilisation of health facilities for delivery by pregnant women living with HIV/AIDs and an impediment to prevention of mother to child (PMTCT) of HIV. Greater comprehension of contextual factors that specifically reduce HIV-positive women's access to maternity care is essential. This paper reviews the existing research literature on AIDS stigma experienced by HIV positive pregnant women in Nigeria with the objective of documenting the current status of research, highlighting major findings and identifying key gaps remaining.

Methods Fifteen publications were identified after a structured search of six electronic databases for published literature between 2000 and 2016 that potentially contained data on HIV-related stigma, utilisation of skilled birth attendants and delivery outcomes. We used the method of meta-synthesis to summarise the findings from the qualitative studies.

Results Stigmatisation experienced includes exclusion from maternity services,termination of appointment, abuse, disrespect, maltreatment, negative attitudes and hostility amongst others. There is increasing concern about health care workers reluctance to care for and treat HIV positive pregnant women. This is further exacerbated by weak health systems and poor legal and ethical framework. HIV positive pregnant women are reluctant to deliver their babies in a health facility with a skilled attendant due to the risk being labelled HIV positive. This avoidance could contribute to obstetric complications and avoidable maternal deaths.

Conclusion Interventions should be introduced to reduce HIVrelated stigma. Training health workers on the elements of psychosocial care and avoiding stigmatising behaviour is important. Research exploring linkages between HIV related stigma and maternity services uptake are largely missing and need to be prioritised. In addition, more research is needed to advance conceptual understanding of stigma within the cultural context of Nigeria.
P4.42 KAP STUDY AND PREVALENCE OF HIV AND SYPHILIS IN HONDURA'S PRIVATE FREEDOM PERSONS

Freddy Tinajeros. Independent Consultant, La Paz, Bolivia

\subsection{6/sextrans-2017-053264.539}

Introduction According to the STI/HIV/AIDS Department's has recorded a total of 32573 cases Accumulated of which 22737 are cases of Advanced Infection and 9836 are asymptomatic HIV. The only previous study of persons deprived of liberty (PPL) was in 1999, which included three cities in Honduras and 2095 people were recruited, the majority of which were men. The prevalence of syphilis was $1.8 \%$.

Methods A cross-sectional study, which used systematic random sampling by clusters, each penal centre gave a proportional share of its population. The universe of persons deprived of liberty consisted of 5813 men (3,531 TGUs and 2,282 SPS) and 430 women (346 TGUs and 84 SPS), who make a total of 6159 . The protocol was approved by the Biomedical Research Committee of the National Autonomous University of Honduras (CEIB).

Results Regarding HIV sero-prevalence, 1.9\% (95\% CI, 0.84.2) of HIV-infected men compared to $0.6 \%$ (CI 95\%, 0.0 3.4) of women, however, men Of SPS 2 in 3.3\% (IC 95\%, 0.9-8.2) of HIV compared to $1.0 \%(95 \% \mathrm{CI}, 0.1-3.5)$ in TGU men, whereas TGU 2 women were $0.8 \%$ (95\% CI, 0.0 4.5 ) and women of SPS $0.0 \%$ (95\% CI, 0.0-8.8), ie no cases were found in the study sample, however this does not mean that there is no HIV in them. The incidence of syphilis (using as a criterion RPR posi2vo confirmed by TPPA) was $1.5 \%$ (95\% CI, 0.3-4.3) in TGU men compared to 3.3\% (95\% CI, $0.9-8.2)$ in SPS men, While in TGU women it was $0.8 \%$ (95\% CI, 0.0-4.5) compared to 7.5\% (CI 95\%, 1.6-20.4) of SPS women.

Conclusion HIV prevalence is higher in San Pedro Sula than in Tegucigalpa, as is the incidence of syphilis, but the highest of all is the prevalence of cumulative syphilis, which implies that there is unprotected sex and high risk. Also, while most know where to perform the HIV test only half were performed and the syphilis test less than a third. One-third of men and women suffered from discrimination and PPL discrimination, with verbal attacks (threats, scoldings, humiliations) among the $2 \mathrm{p}$ of attacks, and less than one-twentieth reported this discrimination.

\section{P4.43 SOCIO-ECONOMIC STATUS OF MEN ENGAGING IN AGE DISPARATE SEX: A SECONDARY ANALYSIS OF NATIONALLY REPRESENTATIVE DATA IN SOUTH AFRICA}

${ }^{1}$ Gavin George, 'Sean Beckett, ${ }^{2}$ Brendan Maughan-Brown, ${ }^{3}$ Meredith Evans. ${ }^{1}$ Health Economics and HIVIAIDS Research Division, University of Kwazulu-Nata, Durban, South African Republic; ${ }^{2}$ Southern Africa Labour And Development Research Unit (SALDRU), Department of Economics, University, Cape Town, South African Republic; ${ }^{3}$ Department of Anthropology, York University, Toronto, Canada

\subsection{6/sextrans-2017-053264.540}

Introduction Studies have highlighted the increased risk in contracting HIV for young women who engage in age disparate sexual partnerships. However, there is a dearth of evidence about the socioeconomic profile of men who engage in these types of sexual partnerships. This study focuses on men who engage in age disparate sex and specifically whether there are socioeconomic-status (SES) asymmetries between those 